\title{
DÜBLIN
}

Technological University Dublin

ARROW@TU Dublin

\section{The influence of correlation on the extreme traffic loading of bridges}

\author{
Eugene J. OBrien \\ University College Dublin \\ Bernard Enright \\ Technological University Dublin, bernard.enright@tudublin.ie
}

A. T. Dempsey

Roughan ODonovan Innovative Solutions

Follow this and additional works at: https://arrow.tudublin.ie/engschcivcon

Part of the Civil Engineering Commons, and the Structural Engineering Commons

\section{Recommended Citation}

OBrien, E. J., Enright, B. and Dempsey, A. T. (2010), 'The influence of correlation on the extreme traffic loading on bridges', In: D. M. Frangopol, R. Sause, and C. S. Kusko, eds. Fifth International Conference on Bridge Maintenance, Safety and Management, Philadelphia.

This Conference Paper is brought to you for free and open access by the School of Civil and Structural Engineering at ARROW@TU Dublin. It has been accepted for inclusion in Conference papers by an authorized administrator of ARROW@TU Dublin. For more information, please contact arrow.admin@tudublin.ie, aisling.coyne@tudublin.ie, gerard.connolly@tudublin.ie.

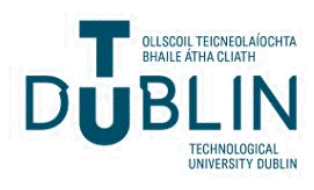




\title{
The influence of correlation on the extreme traffic loading of bridges
}

\author{
E.J. OBrien \\ University College Dublin, Dublin, Ireland
}

B. Enright

Dublin Institute of Technology, Dublin, Ireland

T. Dempsey

Roughan ODonovan Innovative Solutions, Dublin, Ireland

\begin{abstract}
Accurate traffic loading models based on measured data are essential for the accurate assessment of existing bridges. There are well-established methods for the Monte Carlo simulation of single lanes of traffic, and this can easily be extended to model the loading on bridges with two independent streams of traffic in opposing directions. However, a typical highway bridge will have multiple lanes in the same direction, and various types of correlation are evident in measured traffic. This paper analyses traffic patterns using multi-lane WIM data collected at two European sites. It describes an approach to the Monte Carlo simulation of this traffic which applies variable bandwidth kernel density estimators to empirical traffic patterns of vehicle weights, gaps and speeds. This method provides a good match with measured data for multi-truck bridge loading events, and it is shown that correlation has a small but significant effect on lifetime maximum load effects.
\end{abstract}

\section{INTRODUCTION}

Much work has been done on modeling bridge loading due to two-lane same-direction traffic. In the work by Nowak (1993), a number of simplifying assumptions were made - for example that one in 15 heavy trucks has another truck side-by-side, and that for one in 30 of these multiple truck events, the two trucks have perfectly correlated weights. A heavy truck was defined as one with a gross vehicle weight (GVW) in the top $20 \%$ of measured truck weights. As Kulicki et al. (2007) note, the assumptions used were based on limited observations, and the assumptions on weight correlation were entirely based on judgment, as almost no data were available. Moses (2001) presents a simple traffic model for estimating multiple presence probabilities as a function of average daily truck traffic (ADTT), and then selects conservative values, some being based on subjective field observations, for calibrating load factors for bridge assessment. Sivakumar et al. (2007) refine the definition of side-by-side events to include two trucks with headway separation of $\pm 18.3 \mathrm{~m}(60 \mathrm{ft})$, and also consider the influence of the bridge length. Sivakumar et al. (2008), citing Gindy \& Nassif (2006a), extend this further by classifying multiplepresence events as side-by-side, staggered, following or multiple. They present statistics, derived from weigh-in-motion (WIM) measurements, for the frequency of occurrence of these events for different truck traffic volumes and bridge spans. They de- scribe a method for estimating site-specific bridge loading which uses multiple-presence probabilities calculated either directly from WIM data or estimated from traffic volumes using reference data collected at other sites. It is assumed, surprisingly enough. that the GVW distribution is the same in both lanes, and that there is no correlation between weights in adjacent lanes.

In the development of the Eurocode for bridge loading (EC1 2003), characteristic load effects were estimated by extrapolating directly from results for measured traffic, and also by extrapolating from Monte Carlo simulation of traffic, with each lane being simulated independently (Bruls et al. 1996; Dawe 2003; O'Connor et al. 2001).

Croce \& Salvatore (2001) present a theoretical stochastic model based on a modified equilibrium renewal process of vehicle arrivals on a bridge and note that while existing numerical models are particularly efficient when single-lane traffic flow is considered, they are unsatisfactory for multi-lane traffic, and have often employed drastic simplifications. In their model, convolution is used to combine load effect distributions for traffic in multiple lanes.

This study is based on WIM data collected at two European sites. A detailed analysis of the data reveals that for groups of adjacent vehicles in both lanes, there are patterns of correlation and interdependence between vehicle weights, speeds and intervehicle gaps. A Monte Carlo simulation model has been developed for evaluating bridge loading due to 
traffic in two same-direction lanes. This simulation seeks to reproduce the sometimes subtle patterns of correlation that are evident in measured traffic while also adding an element of randomness so as to vary the loading. This study focuses on short to medium span bridges, up to $45 \mathrm{~m}$ long, where free-flowing traffic with dynamics is taken to govern (Bruls et al. 1996; Flint \& Jacob 1996).

\section{WIM DATA}

The WIM data used as the basis for this study were collected at two sites - at Woerden in the Netherlands, and at Sedlice in the Czech Republic. Table 1 summarizes the WIM data sets used.

As can be seen from the GVW distributions for each lane in the Netherlands in Figure 1, there are significant differences between the two lanes, with a much higher proportion of light vehicles in the fast lane (Fig. 1a) and the same is true in the Czech data. In the Netherlands, there is a much higher proportion of extremely heavy vehicles in the slow lane (Fig. 1b) which is important for bridge loading.

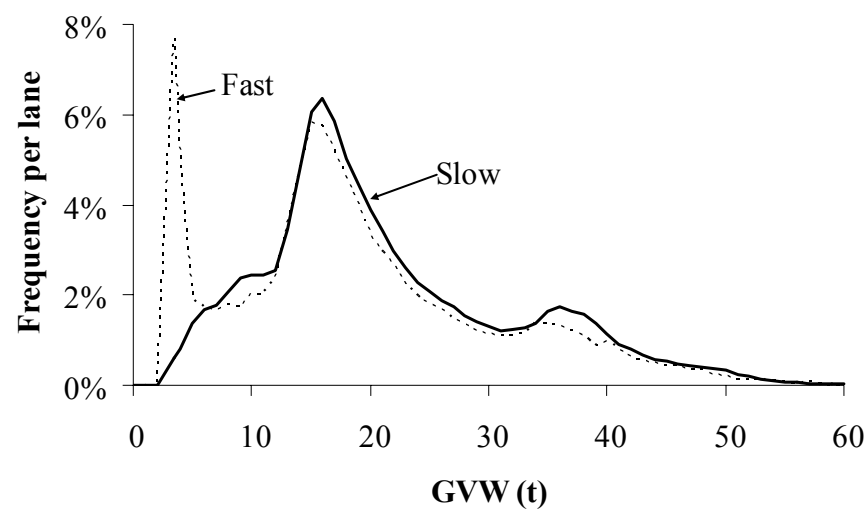

(a) Netherlands up to 60 t [132 kips].

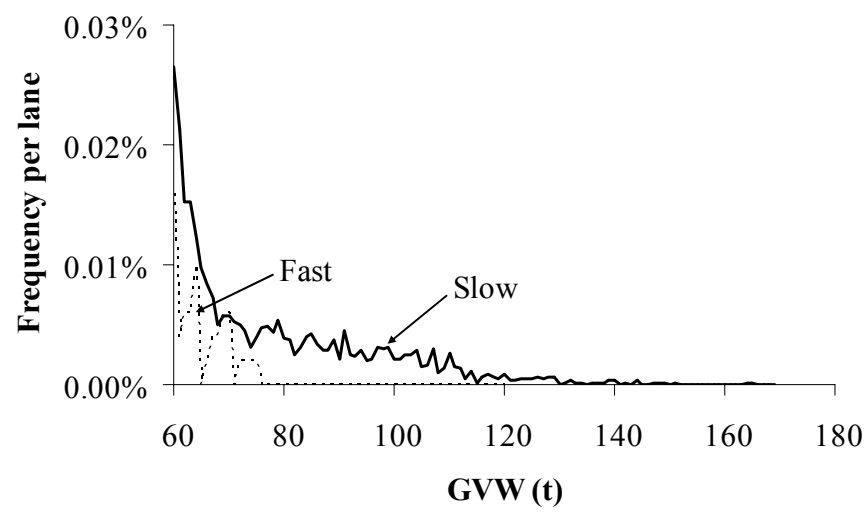

(b) Netherlands over $60 \mathrm{t}$.

Figure 1. GVW distributions.
Table 1. Summary of WIM data.

\begin{tabular}{|c|c|c|c|c|}
\hline Country & Netherl & ands & Czech $\mathrm{F}$ & Republic \\
\hline \multirow[t]{2}{*}{ Time period } & \multicolumn{2}{|c|}{$\begin{array}{l}\text { Feb } 2005 \text { to } \\
\text { June } 2005\end{array}$} & \multicolumn{2}{|c|}{$\begin{array}{l}\text { May } 2007 \text { to } \\
\text { May } 2008\end{array}$} \\
\hline & $\begin{array}{l}\text { Slow } \\
\text { lane }\end{array}$ & $\begin{array}{l}\text { Fast } \\
\text { lane }\end{array}$ & $\begin{array}{l}\text { Slow } \\
\text { lane }\end{array}$ & $\begin{array}{l}\text { Fast } \\
\text { lane }\end{array}$ \\
\hline Total trucks & 596568 & 49980 & 684345 & 45584 \\
\hline $\mathrm{ADTT}^{* *}$ & 6545 & 557 & 4490 & 261 \\
\hline Maximum GVW (t ) & 166 & 75 & 129 & 128 \\
\hline (kips) & 365 & 165 & 284 & 282 \\
\hline No. over 60 t [132 kips] & 1680 & 36 & 322 & 54 \\
\hline No. over 100 t [220 kips] & 238 & 0 & 10 & 2 \\
\hline
\end{tabular}

* Average daily truck traffic per lane on week days.

\section{CORRELATIONS IN MEASURED DATA}

\subsection{Vehicle weights}

For short to medium span bridges, loading events featuring one truck in each lane (either side-by-side or staggered) are particularly important. To assess if there is any dependence between the weights of these vehicles, each fast-lane truck in the measured data is notionally paired with the nearest truck in the slow lane, and the gap is measured in seconds between the front axles of the two vehicles. At both sites, most fast-lane trucks are within 2 seconds of a slow-lane truck $-75 \%$ in the Netherlands and $72 \%$ in the Czech Republic. The average GVW of the truck in the fast lane and of the nearest truck in the slow lane are plotted against the inter-lane gap for the Netherlands in Figure 2. There is a significant peak in the fast lane GVW when the gap is around zero - i.e. when the trucks are very close - and a similar pattern is evident in the Czech Republic. It appears that a heavy truck in the fast lane tends to be associated with a nearby truck in the slow lane, i.e. it is passing another truck.

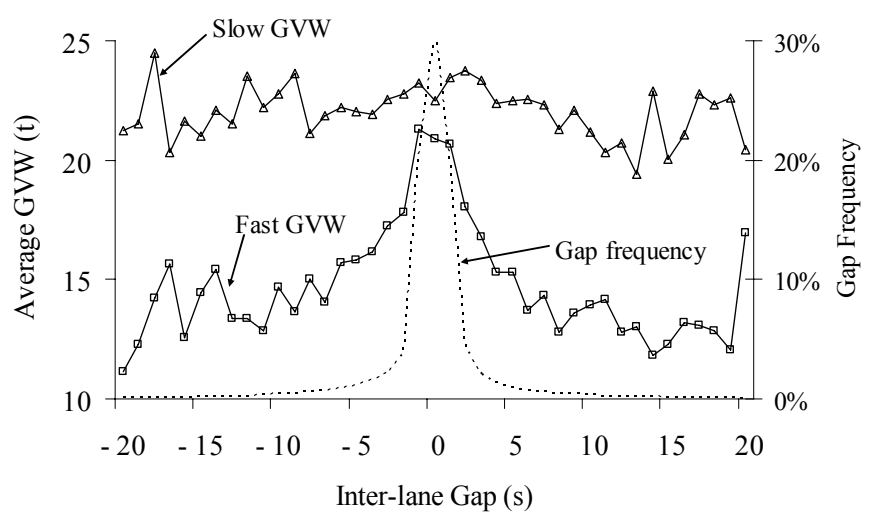

Figure 2. Inter-lane GVW correlation, the Netherlands.

\subsection{Gaps and speeds}

It is well established that the distribution of samelane gaps between vehicles varies with traffic flow 
rate (O'Brien \& Caprani 2005); in general gaps are less for higher flows. It is evident from the WIM data used here that there is also some slight dependence between gaps and GVW, and that successive gaps are not independent. At both sites, the axle to axle gap observed behind vehicles tends to increase as the GVW increases. This can be attributed partly to driver behaviour, perhaps greater overhang (axle to bumper) distances, and also to the fact that many trucks in excess of the normal legal weight limit are followed by escort vehicles. The idea that successive gaps are not independent is reasonably intuitive. The platooning effect commonly observed on highways means that smaller gaps tend to occur in groups.

As might be expected, there is a tendency for heavier vehicles to travel at slightly lower speeds, although most extremely heavy vehicles are travelling at around $80 \mathrm{~km} / \mathrm{h}$ which would be regarded as a normal highway speed for any truck. Speeds of successive vehicles in the same lane show a relatively high degree of correlation when the inter-vehicle gaps are small, with an average coefficient of correlation for both sites of 53\% when the gap is less than 2 seconds. This drops to $15 \%$ when the gap is more than 2 seconds.

\section{SIMULATION OF TRAFFIC}

It is evident from the foregoing that there are discernible patterns in the measured traffic that may be significant for bridge loading. Using measured traffic to calculate a distribution of load effects and then extrapolating from this to lifetime maxima implicitly incorporates the patterns in the traffic, but suffers from high uncertainty due to the extrapolation process. Variation in results from extrapolation of up to $33 \%$ have been reported by Gindy \& Nassif (2006b), and up to $20 \%$ for the estimation of characteristic load for the Eurocode (Dawe 2003). The approach used here is to build a Monte Carlo simulation model that incorporates the patterns and then to run the simulation for a sufficiently long time period to avoid the problems associated with extrapolation.

The spatial layout of vehicles on a two-lane bridge can be described by three gap distributions in-lane gaps for each of the two lanes and inter-lane gaps. The standard approach to simulating random variables is to generate values from the required distributions. In this case, the three gap distributions cannot be simulated independently - for example generating random values from the two in-lane gap distributions will position vehicles in each lane, and this automatically determines the inter-lane gap distribution. For bridge loading, it might be reasonable to assume that the slow-lane and inter-lane gaps are more important than the fast-lane gaps. On this basis the slow-lane and inter-lane gaps can be simulated directly from the distributions, and a good match be- tween observed and simulated gaps can be obtained. However, the simulation of the fast-lane gaps is completely wrong, with the platooning effect in that lane being lost in the simulation.

In order to build a conventional simulation model for two same-direction lanes, all significant patterns in the measured data must be identified and quantified in some way that can be incorporated into the simulation. It is possible to build a reasonably accurate model in this way, but the process is very sitespecific and time-consuming and the model needs to be carefully calibrated. Extending such a model from two to three or more lanes would be very challenging. An alternative multi-dimensional smoothed bootstrap approach is adopted here which avoids many of the difficulties associated with the conventional approach, and in principle can quite easily be extended to more than two lanes.

The principle of bootstrapping is to repeatedly draw random samples from the observed data (Efron \& Tibshirani 1993). In this case, the samples used are "traffic scenarios", with each scenario consisting of between five and eight slow-lane trucks in succession, with any adjacent fast-lane trucks. In preparation for simulation, the WIM data are analysed and all scenarios are identified. The parameters recorded for each scenario are flow rate, gaps, GVWs and speeds. The flow rate is represented by the number of slow-lane trucks in the current hour, rounded to the nearest 10 trucks/hour. The gaps needed to define the scenario are the gaps within each lane, and one inter-lane gap which positions the first fast-lane truck relative to the leading slow-lane truck in the scenario, as shown in Figure 3.

The number of parameters needed to describe a single scenario (i.e. the dimensionality of the problem) varies with the size of the scenario, but in the typical scenario shown in Figure 3, a total of 21 different parameters are needed - the GVWs and speeds of seven trucks, six gap values and a flow rate. Correlations between parameters are implicitly included in each scenario.

The aim in setting up the scenarios is to keep them reasonably small so as to maximise the variability in the simulation, but also to have them large enough to capture patterns that may be significant for bridge loading. In order to preserve any significant groups of heavy vehicles in the slow lane, the first and last slow-lane trucks are required to be less than $30 \mathrm{t}$ [66 kips]. Hence, starting from a truck less than $30 \mathrm{t}$, trucks are included until another less than $30 \mathrm{t}$ is found. The last truck in each scenario becomes the first in the next scenario. In order to provide greater coverage of different scenarios, four scans are made through the WIM data with the minimum scenario size varying from five slow-lane trucks for the first scan up to eight for the last scan. 


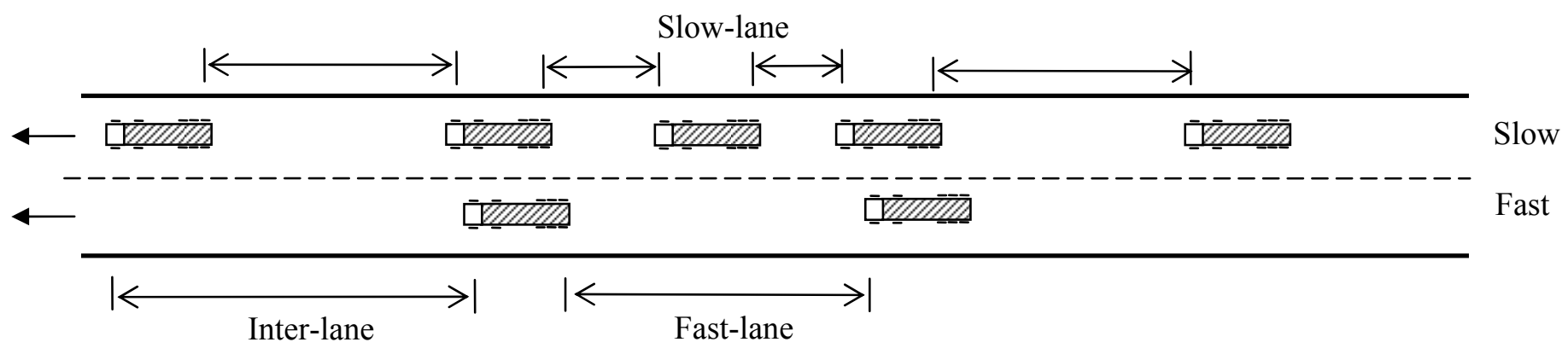

Figure 3. Traffic scenario.

In the simulation process, a flow rate is determined for the time of day, based on average measured values for all weekdays. A scenario is selected at random from all scenarios corresponding to this flow rate. For a given traffic flow rate, each scenario has an equal probability of selection, and this means that the measured relative frequencies of the parameters defining the scenarios are reproduced in the simulation. The number of different scenarios for a given flow rate depends on the quantity of measured WIM data, but at both sites there are in excess of 20000 scenarios for each of the commonly observed flow rates. The trucks in the selected scenario are added to the stream of traffic, the time is advanced, and another scenario is selected. The scenarios are joined together by overlapping the last truck of the previous scenario with first truck in the new scenario and then discarding the latter. As noted already, the overlapping trucks are all less than $30 \mathrm{t}$.

This bootstrap process would be expected to produce bridge loading very similar to the measured traffic. The measurements have been collected over a number of months, but in order to estimate lifetime maximum bridge loading, many years of traffic must be simulated. A key part of this process is to extend the simulation to incorporate scenarios that have not been directly observed. Of particular interest is the modeling of vehicles heavier than, and with more axles than, any measured vehicles. Different gap combinations than those observed also need to be allowed to occur. Variations from the observed scenarios are introduced in a number of ways. Each time a scenario is selected in the simulation, the GVWs, gaps and speeds that define it are modified using variable-bandwidth kernel density estimators, as described in the following section. When a GVW has been selected for a particular vehicle, the number of axles is randomly chosen from the measured distribution for that weight. The axle spacings, and distribution of the GVW to individual axles, are also generated randomly from measured distributions for vehicles with different numbers of axles. The approach used for vehicle modeling is described in more detail by Enright \& O'Brien (2009b).

\subsection{Kernel density estimators}

The term "kernel density estimator" describes the use of kernel functions to provide a better estimate of a probability density function from sample data (Scott 1992). A simple histogram gives an estimate of the density at discrete points, but is influenced by the choice of the bin size and origin. Replacing each data point by a kernel function and summing these functions gives a better estimate. Different kernel functions can be used - they are typically symmetric unimodal functions such as the Normal density function. In Monte Carlo simulation, for each random variable, some estimate of its probability density is required. This estimate can be a parametric fit to the data or some non-parametric density. One nonparametric method is to use interpolation on the empirical cumulative distribution, but using a kernel density estimate gives a better coverage of the design space which is important for generating traffic loading scenarios that will be critical for bridges. As Hormann \& Leydold (2000) point out, the "smoothed bootstrap" method - re-sampling the observed data and adding some noise - is the same as generating random variates from the kernel density estimate, but without needing to compute the estimated density. In this study, the smoothed bootstrap is applied to three variables - GVW, gaps and speeds. Each value $x_{i}$ taken from the observed traffic scenarios is modified by adding some noise:

$X_{i}=x_{i}+K\left[h\left(x_{i}\right)\right]$

where $K$ is a kernel function, centered at zero with a variable bandwidth $h$ which depends on the value of $x_{i}$. For each random variable being modeled, a suitable bandwidth must be chosen - if the bandwidth is too small, not enough variability will be introduced to the empirical data, whereas too large a bandwidth will oversmooth the data. 


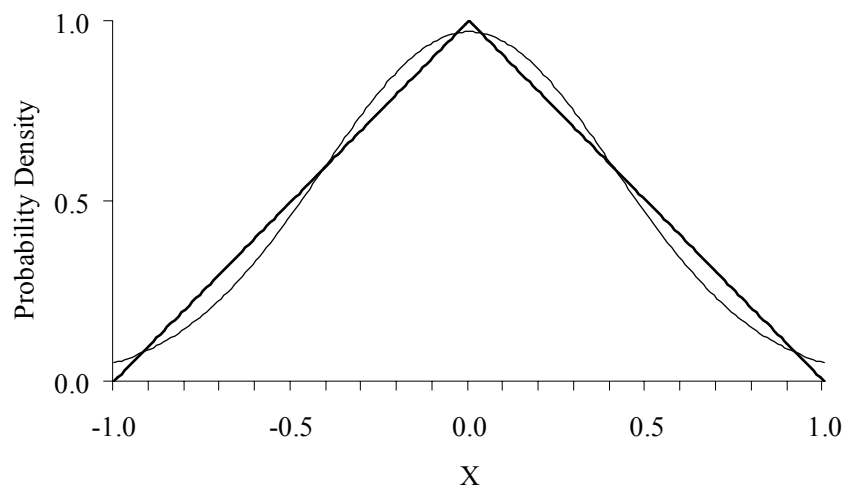

Figure 4. Normal and triangle kernel functions.

Scott (1992) suggests that the choice of which kernel function to use is much less important than the choice of bandwidth. A triangle kernel is used here for gaps because its boundedness is useful at very small gaps, and a Normal kernel is used for GVW. Equivalent Normal and triangle kernel functions are shown in Figure 4. The bandwidth of the triangle kernel in this example is 1.0 , and the bandwidth (standard deviation) for the equivalent Normal kernel is 0.411 (Scott 1992).

The modeling of the upper tail of the GVW distribution is critically important, and O'Brien et al. (2009) describe a method which involves fitting the tail of a Normal distribution to the upper tail of the measured GVW distribution to allow for interpolation between relatively sparse data values and for extrapolation to higher GVW values that are likely to be encountered during the lifetime of a bridge. Using a Normal kernel with a suitable variable bandwidth achieves similar results. The chosen bandwidth formulations for the different parameters are summarized in Table 2.

Table 2. Kernel bandwidths.

\begin{tabular}{lll}
\hline Variable $(x)$ & Kernel & Bandwidth \\
\hline Slow-lane GVW (t) $*$ & Normal & $0.08\left(x^{2}\right) / \operatorname{Max}(x)$ \\
Fast-lane GVW $(\mathrm{t})$ & Normal & $0.065\left(x^{2}\right) / \operatorname{Max}(x)$ \\
Slow-lane gap $(\mathrm{s})$ & Triangle & $\operatorname{Min}(0.2 x, 0.4)$ \\
Fast-lane gap $(\mathrm{s})$ & Triangle & $\operatorname{Min}(0.3 x, 0.6)$ \\
Inter-lane gap $(\mathrm{s})$ & Triangle & $\operatorname{Min}(0.08|x|, 0.16)$ \\
Slow-lane speed $(\mathrm{km} / \mathrm{h})$ & Triangle & 0.6 \\
Fast-lane speed $(\mathrm{km} / \mathrm{h})$ & Triangle & 1.0 \\
\hline
\end{tabular}

* For GVWs, $\operatorname{Max}(x)$ is the site-specific maximum observed GVW per lane

\section{VALIDATION}

In order to assess the simulation models, comparison is made between bridge loading by measured traffic and by simulated traffic on bridges of different lengths $-15,25,35$ and $45 \mathrm{~m}$. For the measured traffic, bridge load effects are calculated by moving the measured stream of traffic over each bridge. For convenience, these are referred to in the following as "measured" load effects. Daily maximum values are calculated for three load effects - mid-span bending moment on a simply supported bridge (LE1), support shear at the entrance to a simply supported bridge (LE2), and for bridges which are $35 \mathrm{~m}$ or longer, hogging moment over the central support of a two-span continuous bridge (LE3).

As well as calculating the overall daily maxima, different loading event types are analysed. It is evident that the two most important loading events in the lifetime maximum loading for the spans considered are the one-truck event (" $1+0$ ") and the twotruck event with one truck in each lane ("1+1"). As the span increases, four other event types are included in the comparison of the different simulation methods - the $1+2,2+1,2+0$ and $2+2$ events, where " $i+j$ " indicates $i$ and $j$ truck(s) in the slow and fast lanes respectively. These are less onerous for the spans considered at the two sites, but could become significant at longer spans or at other sites with different traffic characteristics. The $1+2$ and $2+1$ events are considered for spans of $25 \mathrm{~m}$ and longer, the $2+0$ event for the 35 and $45 \mathrm{~m}$ spans, and the $2+2$ event for the $45 \mathrm{~m}$ span.

To assess the effects of correlation, an uncorrelated simulation model was also developed in which GVWs, slow-lane gaps, and speeds are drawn independently for each truck from the observed distribution in the appropriate lane. Gap distributions are measured at 25 different flow rates, and the distribution appropriate to the flow (time of day) is used. For a site-specific percentage of slow-lane trucks, a fast-lane truck is generated and positioned relative to the slow-lane truck by drawing a value from the inter-lane gap distribution. As noted earlier, this does not model the fast-lane gaps well.

For comparison purposes, the two simulation models - smoothed bootstrap and uncorrelated were run for 2000 days, and the simulated and measured results plotted on Gumbel paper (Ang \& Tang 1975). An example is shown in Figure 5 for $1+1$ events on a $25 \mathrm{~m}$ bridge in the Netherlands, and this illustrates that the smoothed bootstrap gives a significantly better fit to the measured data.

An analysis of all spans, load effects and event types described above shows that in general the smoothed bootstrap gives a better fit to the measured data for multi-truck events. For one-truck events, both methods perform equally well. 


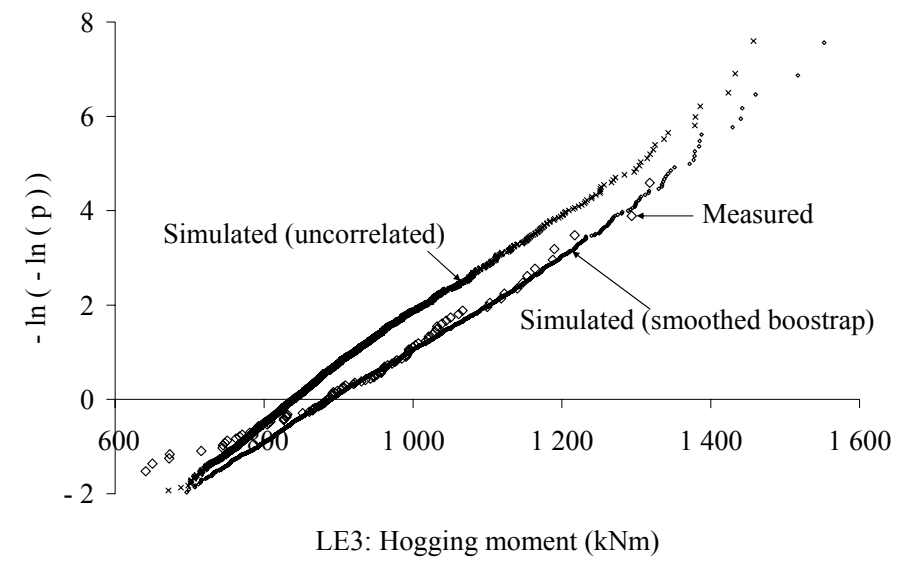

Figure 5. Simulated and measured daily maximum load effects.

\section{RESULTS}

To see what effect the different modeling assumptions have on the characteristic maximum loading, both methods were used to simulate 2500 years of traffic. In the Eurocode for bridge loading (EC1 2003 ), the value with a $5 \%$ probability of exceedance in 50 years is specified for design which is approximately the value with a return period of 1000 years. The focus in the AASHTO design code is on the mean 75-year maximum (Nowak 1995), and the effects of the different models on this are also calculated.

Lateral distribution is accounted for by applying different lane factors to truck weights in the fast lane. These factors are based on finite element analyses carried out by the authors (Enright \& O'Brien 2009a). For bending moments on bridges with high lateral distribution, the factor is 1.0 (i.e. no reduction), and 0.45 for low distribution. Maximum shear at the supports occurs when trucks are close to the support, and there is less opportunity for lateral distribution. In this case, a factor of 0.45 represents high distribution, and 0.05 is low.

Sample results are plotted in Figure 6 which shows simulated annual maxima on a $45 \mathrm{~m}$ bridge in the Netherlands with high lateral distribution. Four event types are shown - one truck in the slow lane $(1+0)$, one truck in each lane $(1+1)$, two trucks in the slow lane $(2+0)$, and one truck in the slow lane with two trucks in the fast lane $(1+2)$. For the $1+0$ event, both models give the same results, but for events involving two or more trucks there are significant differences between the two simulation models, with the smoothed bootstrap method giving more conservative results than the uncorrelated model. The curves are reasonably parallel for the $1+1$ and $2+0$ events, but in the case of the $1+2$ event, the curves converge as the return period increases. It can be seen that in this example, the $1+1$ event governs at the 1000-year return level.

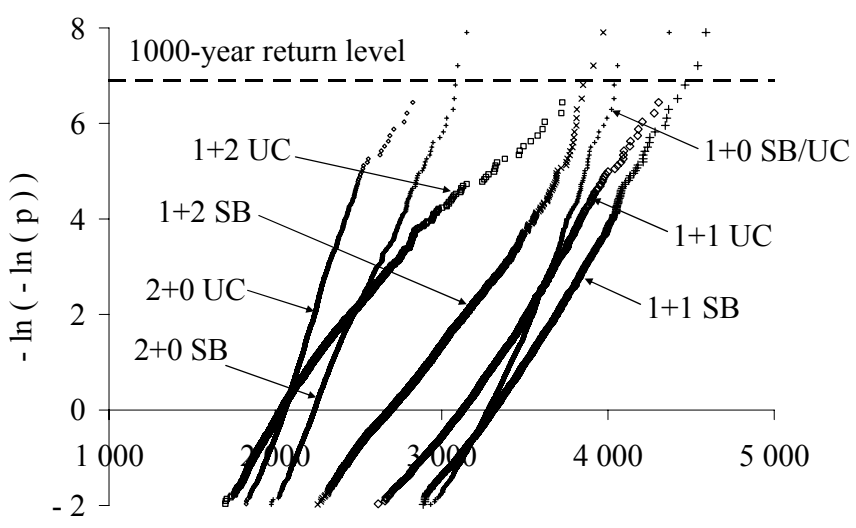

LE3: Hogging moment $(\mathrm{kNm})$

Figure 6. Annual maxima - smoothed bootstrap (SB) and uncorrelated model (UC).

The increases in characteristic maximum load effects due to correlation in models were calculated for the four spans and three load effects considered at each site. Confidence intervals estimated using a parametric bootstrap indicate that differences between $-3.4 \%$ and $+3.4 \%$ for the 1000 -year values are not significant (at 99\% confidence). For the 75-year values, the corresponding confidence interval is between $-1.9 \%$ and $1.9 \%$. Correlation effects were found to account for an increase in 1000-year loading of up to nearly $8 \%$, with typical values of around $5 \%$, particularly when lateral distribution is high. For the mean 75-year maximum, correlation accounted for increases of up to $7 \%$. The types of loading event that govern the characteristic maximum at the 1000-year return level were also identified. In some cases, just one event type is clearly dominant (i.e. either the $1+0$ or the $1+1$ event), but in other cases there is a mixture of both event types, and for the longer spans ( 35 and $45 \mathrm{~m}$ ) in the Czech Republic, some simulated $1+2$ events produce bending moments close to the characteristic values.

A closer examination of the events in the simulations that produce the characteristic 1000-year loads shows that for bridges with low lateral transfer, the critical loading event for bending moment is typically an extremely heavy vehicle in the slow lane $(80 \%$ to $90 \%$ of the 1000 -year GVW), with a standard vehicle (in the range 30 to $40 \mathrm{t}$ [66 to $88 \mathrm{kips}$ ]) in the fast lane - similar to Turkstra's rule (Naess \& Røyset 2000). For bending moment in bridges with high lateral distribution, it is a very heavy vehicle $(60 \%$ to $80 \%$ of 1000 -year GVW) in the slow lane with a moderately heavy vehicle (50 to $60 \mathrm{t}$ [110 to 132 kips]) in the fast lane - a variation on Turkstra's rule. For shear at the supports, lateral distribution tends to be low, and the dominant event type is usually a single extremely heavy truck in the slow lane ( $75 \%$ to $95 \%$ of the 1000 -year GVW). 


\section{CONCLUSIONS}

There are subtle patterns of correlation evident in measured traffic data. This inter-dependence between weights, speeds and inter-vehicle gaps for adjacent trucks affects the estimation of lifetime maximum bridge loading. While it may be possible to model this dependence reasonably well using conventional Monte Carlo simulation techniques, an alternative multi-dimensional smoothed bootstrap approach is presented here which re-samples observed traffic scenarios and uses kernel functions to introduce additional variation. The traffic scenarios are defined so as to capture patterns that may be significant for bridge loading, and to maximise variability in the simulation. The method is relatively simple to implement for any new site, and could be extended to three or more lanes. It is effectively the same as sampling from empirical distributions (for GVW, gaps and speed), but with correlation and some additional smoothing and randomness. It potentially could be used to model congested or partly congested traffic, if sufficient data were available. The choice of bandwidth for the kernel smoothing functions is somewhat arbitrary, although results for characteristic bridge loading are, within reason, not too sensitive to this choice.

The model presented provides a better fit to measured data across the range of key loading event types than is obtained with a model which does not include any correlation effects. The effects of correlation on characteristic maximum loading may be as high as $8 \%$ for the range of bridge spans considered. The uncorrelated model, which is somewhat easier to implement, is less accurate and is nonconservative.

\section{ACKNOWLEDGEMENTS}

The authors gratefully acknowledge the support of the European $7^{\text {th }}$ Framework Project, ASSET.

\section{REFERENCES}

Ang, A. H.-S. \& Tang, W. H. (1975), Probability concepts in engineering planning and design, New York: Wiley.

Bruls, A., Croce, P., Sanpaolesi, L. \& Sedlacek, G. (1996), 'ENV1991 - Part 3: Traffic Loads on Bridges; Calibration of Load Models for Road Bridges', Proceedings of IABSE Colloquium, Delft, The Netherlands, IABSE-AIPC-IVBH, 439 - 453.

Croce, P. \& Salvatore, W. (2001), 'Stochastic model for multilane traffic effects on bridges', Journal of Bridge Engineering, ASCE, 6 (2), 136-143.

Dawe, P. (2003), Research Perspectives: Traffic loading on highway bridges, London: Thomas Telford.

EC1. (2003), Eurocode 1: Actions on structures, Part 2: Traffic loads on bridges, Brussels: European Standard EN 19912:2003: European Committee for Standardization, TC250.
Efron, B. \& Tibshirani, R. (1993), An Introduction to the Bootstrap Chapman and Hall/CRC.

Enright, B. \& O'Brien, E. J. (2009a), 'Maximum lifetime traffic loading scenarios on short and medium span bridges', $S u b$ mitted for publication.

Enright, B. \& O'Brien, E. J. (2009b), 'Monte Carlo Simulation of extreme traffic loading on short and medium span bridges', Submitted for publication.

Flint, A. R. \& Jacob, B. (1996), 'Extreme Traffic Loads on Road Bridges and Target Values of Their Effects for Code Calibration', Proceedings of IABSE Colloquium, Delft, The Netherlands, IABSE-AIPC-IVBH, 469 - 478.

Gindy, M. \& Nassif, H. H. (2006a), 'Multiple Presence Statistics for Bridge Live Load Based on Weigh-in-Motion Data', Transportation Research Board 86th Annual Meeting, Washingotn D.C.

Gindy, M. \& Nassif, H. H. (2006b), 'Comparison of traffic load models based on simulation and measured data', Joint International Conference on Computing and Decision Making in Civil and Building Engineering, Montréal, Canada, [online] available from: http://www.icccbexi.ca/html/en/AutIndex.htm\#G, accessed 8 May 2007

Hormann, W. \& Leydold, J. (2000), 'Automatic Random Variate Generation for Simulation Input', Proceedings of the 2000 Winter Simulation Conference, J. A. Joines, R. R. Barton, K. Kang, \& P. A. Fishwick, eds., 675-682, [online] available from: http://citeseer.ist.psu.edu/312173.html, accessed 19 April 2007

Kulicki, J. M., Prucz, Z., Clancy, C. M., Mertz, D. \& Nowak, A. S. (2007), 'Updating the calibration report for AASHTO LRFD code', NCHRP. Washington D.C.: Transportation Research Board.

Moses, F. (2001), 'Calibration of load factors for LRFR Bridge Evaluation', NCHRP Report No. 454. Washington D.C.: Transportation Research Board.

Naess, A. \& Røyset, J. Ø. (2000), 'Extensions of Turkstra's rule and their application to combination of dependent load effects', Structural Safety, 22, 129-143.

Nowak, A. S. (1993), 'Live load model for highway bridges', Structural Safety, 13, 53-66.

Nowak, A. S. (1995), 'Calibration of LRFD Bridge Design Code', Journal of Structural Engineering, ASCE, 121 (8), 1245-1251.

O'Brien, E., Enright, B. \& Getachew, A. (2009), 'Importance of the Tail in Truck Weight Modeling for Bridge Assessment', Journal of Bridge Engineering, ASCE, in print.

O'Brien, E. J. \& Caprani, C. C. (2005), 'Headway modelling for traffic load assessment of short- to medium-span bridges', The Structural Engineer, 83 (16), 33-36.

O'Connor, A., Jacob, B., O'Brien, E. J. \& Prat, M. (2001), 'Report of current studies performed on normal load model of EC1 Part 2. Traffic loads on bridges', Revue Française de Génie Civil, 5 (4), 411-433.

Scott, D. W. (1992), Multivariate density estimation : theory, practice, and visualization, New York: Wiley.

Sivakumar, B., Ghosn, M. \& Moses, F. (2008), 'Protocols for Collecting and Using Traffic Data in Bridge Design', W135, NCHRP. Washington D.C.: Transportation Research Board, [online] available from: http://onlinepubs.trb.org/onlinepubs/nchrp/nchrp_w135.pdf, accessed 2 July 2009

Sivakumar, B., Moses, F., Fu, G. \& Ghosn, M. (2007), 'Legal truck loads and AASHTO Legal Loads for Posting', NCHRP Report 575. 